\section{Stuhltransfer als Therapieoption?}

Bei M. Crohn könnte auch die Zusammensetzung der Darmflora relevant sein. Bei Mäusen verursacht ein bakterielles Ungleichgewicht eine Crohn-typische Entzündung, die auf andere Tiere übertragbar ist. Damit könnte die Stuhltransplantation weiterentwickelt werden, teilt die TU München mit. Forscher transplantierten das Mikrobiom kranker Mäuse in keimfreie. Obwohl die Mausstämme eine erhöhte Crohn-Veranlagung hatten, entwickelten sie erst Symptome, als innen die Bakterien erkrankter Tiere eingepflanzt wurden. Dabei sind nicht einzelne Bakterienarten die Auslöser, sondern dysbiotische Kombinationen.

Gut 2015; online 17. April

\section{Jahre}

sind Patienten alt (median), wenn sie an Morbus Crohn erkranken; die höchste altersspezifische Inzidenz liegt im dritten Lebensjahrzehnt. Hochgerechnet würden in Deutschland 2-3 Mrd.€ pro Jahr für die Versorgung M. Crohn-Kranker aufgewandt, so die Autoren der Leitlinie zu M. Crohn. 64\% der Gesamtkosten seien den indirekten Kosten zuzuordnen. An direkten medizinischen Kosten seien zuletzt zwischen 3500 und $6000 €$ pro Jahr für einen M. Crohn-Kranken errechnet worden.

\section{INFOS IM INTERNET}

www.dccv.de - die Seite der DCCV, der Deutschen M. Crohn/Colitis-ulcerosa-Vereinigung

Vom 19. September bis zum 18. Oktober 2015 findet der Crohn \& Colitis-Tag statt. Infos dazu gibt's im Web unter www.crohn-und-colitistag.de

Speziell für Patienten mit Stoma bietet die Deutsche ILCO, die Deutsche Vereinigung der Stomaträger, Infos und Unterstützung: www.ilco.de

Links zu den deutschen und europäischen Leitlinien: www.dgvs.de und www.ecco-ibd.eu

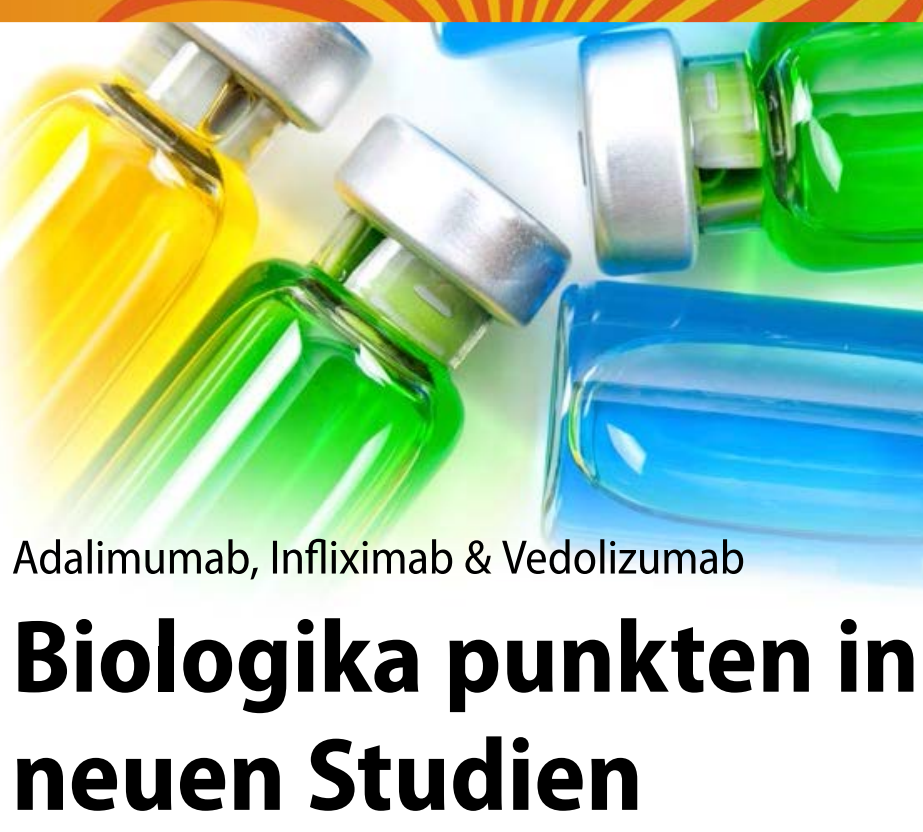

Beim Gastro Update wurden neue Studien zu Biologika bei Morbus Crohn diskutiert.

Bei steroidrefraktärem Verlauf eines Morbus Crohn sollte vor immunsuppressiver Therapie eine Op. als Alternative geprüft werden. Ohne Op.-Indikation bestehe die Indikation zur Therapie mit konventionellen Immunsuppressiva oder AntiTNF-Antikörpern, so Prof. Dr. Volker Groß vom Klinikum St. Marien Amberg beim Gastro Update in Wiesbaden.

Bei höherer Krankheitsaktivität sollte einer Anti-TNF-Therapie, eventuell kombiniert mit einem klassischen Immunsuppressivum, der Vorzug gegeben werden. Auch bei Patienten, die auf systemische Steroide ansprechen, bei Dosisreduktion aber rasch ein Rezidiv entwickeln, sei ein TNF-Blocker, eventuell kombiniert mit einem klassischen Immunsuppressivum indiziert, so Groß.

- Für Adalimumab (Humira ${ }^{\circledR}$ ) liegen Studien vor, nach denen Patienten, die auf eine Induktionstherapie ansprechen, auch langfristig von Adalimumab profitieren. Groß verweist hier auf eine Studie über einen Zeitraum von vier Jahren [1]: Von 329 frühen Respondern auf eine Adalimumab-Induktionstherapie waren etwa 30\% nach vier Jahren noch in Remission, 116 der 329 Patienten zeigten noch eine CDAI-Abnahme von mindestens 100 Punkten. Die Mehrzahl der Patienten in Remission nach einem Jahr verblieben auch nach vier Jahren noch in Remission. In einer anderen Studie hatten M. Crohn-Kranke eine Induktionstherapie mit Adalimumab erhalten, danach Adalimumab oder Pla- cebo. Nach einem Jahr hatten 19\% der Adalimumab-Patienten eine „deep remission“ (CDAI $<150$ plus endoskopische Remission), mit Placebo war das bei keinem Patienten der Fall [2].

- Für Infliximab (Remicade ${ }^{\circledR}$ ) wurde gezeigt, dass bei Patienten, die nach längerer Pause eine Anti-TNF-Therapie wieder aufnehmen, das Fehlen von Antikörpern gegen die Arznei und hohe Medikamenten-Talspiegel günstig für eine erfolgreiche Re-Therapie sind. Von 128 Patienten, die nach im Mittel 15 Monaten wieder Infliximab bekamen, hatten nach 14 Wochen $85 \%$ auf die Therapie angesprochen, nach einem Jahr 70\% und nach über vier Jahren $61 \%$. Wurden bei der ersten Kontrolle Antikörper gegen Infliximab festgestellt, lag die Ansprechrate nach einem Jahr bei 55\%, langfristig bei 39\% [3].

- Vedolizumab (Entyvio ${ }^{\circledR}$ ), ein gegen a4ß7-Integrin gerichteter Antikörper, hemmt die Lymphozytenmigration in den Darm. Nach einer Studie, in der 315 M. Crohn-Patienten mit Versagen einer Anti-TNF-Therapie, aber auch $101 \mathrm{~Pa}-$ tienten ohne vorangehende Anti-TNFTherapie mit Vedolizumab behandelt wurden [4], sprechen Anti-TNF-naive Patienten besser an als Anti-TNF-Versager, so Groß. Jedoch spreche auch ein substanzieller Anteil der Anti-TNFVersager auf Vedolizumab an; es dauere aber länger, bis der Effekt erreicht werde.

(mal)

[1] Aliment Pharmacol Ther 2013; 38: 1236-1247 [2] Clin Gastroenterol Hepatol 2014; 12: 414-422 [3] Clin Gastroenterol Hepatol 2014; 12: 1474-1481 [4] Gastroenterology 2014; 147: 618-627 